\title{
Tunable Narrow-Linewidth Photonic Microwave Generation Using Semiconductor Laser Dynamics
}

\author{
Sze-Chun Chan, Student Member, IEEE and Jia-Ming Liu, Senior Member, IEEE
}

\begin{abstract}
Generation of a broadly tunable narrow-linewidth microwave subcarrier on an optical wave by exploiting the nonlinear dynamics of a semiconductor laser through a proper combination of optical injection and optoelectronic feedback is experimentally demonstrated. The microwave frequency is generated by the period-one oscillation of an optically injected semiconductor laser. It is tuned in the range from 10 to $23 \mathrm{GHz}$ by varying the optical injection strength, and its linewidth can be narrowed by optoelectronic feedback alone. The linewidth is reduced from the range of 40-120 $\mathrm{MHz}$ without stabilization by three orders of magnitude to the range of $10-160 \mathrm{kHz}$ with stabilization through optoelectronic feedback alone. The effect of a small microwave modulation is also investigated. It reduces the linewidth to below the $1-\mathrm{kHz}$ resolution limit of our instrument.
\end{abstract}

Index Terms-Microwave generation, narrow linewidth, nonlinear dynamics, semiconductor laser, tunable.

\section{INTRODUCTION}

G ENERATION of a tunable microwave subcarrier on an optical wave for various photonic and optoelectronic applications has long received great attention [1]-[4]. The simplest approach is direct current modulation of a semiconductor laser with a microwave source. However, due to intrinsic and parasitic constraints, both modulation frequency and modulation amplitude of direct microwave modulation on a semiconductor laser are very limited. Various approaches have been investigated to generate optical waves carrying microwaves using semiconductor lasers without direct modulation. One approach exploits the nonlinear dynamics of a semiconductor laser to generate the microwave internally. The period-one oscillation of a slave laser under strong optical injection is used. By properly varying the injection strength, the frequency detuning, or both, the microwave frequency generated through the period-one oscillation can be tuned continuously over a wide range up to 3 or 4 times the intrinsic resonance oscillation frequency of the slave laser [5]. While broadly tunable, this microwave generally has a relatively large linewidth on the order of 10-100 MHz, hampering its usefulness to many applications that require a narrow linewidth. Using an externally applied microwave that is tuned to the period-one oscillation frequency, this microwave subcarrier can be stabilized by a double-lock technique demonstrated by Simpson and Doft [1]. Using this technique, a microwave subcarrier that is tunable from 9.5 to $17.1 \mathrm{GHz}$ with a linewidth below the instrument resolution limit of $1 \mathrm{kHz}$ is obtained with an optically injected semiconductor laser [1]. Further stabilization of the microwave

Manuscript received January 22, 2004; revised July 26, 2004.

The authors are with the Electrical Engineering Department, University of California, Los Angeles, Los Angeles, CA 90095-1594 USA.

Digital Object Identifier 10.1109/JSTQE.2004.836020 is accomplished through a phase-lock loop to lock the phase of the microwave subcarrier to an external microwave [6]. Another approach uses an optical heterodyne of two lasers to generate the microwave at their difference frequency [2], [3]. In order to stabilize the microwave frequency, one laser is externally modulated and is optically injected into the other laser. The slave laser is then stably injection locked to a sideband of the master laser. Further stabilization can also be done by an optical phase-lock loop [2]. An optical wave carrying a microwave frequency of 4-60 GHz with a minimal linewidth of less than $1 \mathrm{kHz}$ is demonstrated in such a system [4].

All of the above-mentioned methods require an external microwave source as a frequency reference to stabilize the microwave subcarrier frequency. For many applications, it is desirable to eliminate the external microwave source. In this work, we experimentally investigate the possibility of using the nonlinear semiconductor laser dynamics, without any external microwave source, to reduce the linewidth of the tunable microwave subcarrier generated by the period-one oscillation of an optically injected semiconductor laser. In particular, stabilization of the microwave frequency in an optical injection system subject to optoelectronic feedback is examined. Optical injection is responsible for the generation of the tunable microwave subcarrier through period-one oscillation under proper injection conditions, as described in [1] and [5]. Optoelectronic feedback, instead of an external microwave source, is employed for frequency stabilization.

The optoelectronic feedback loop resembles an optoelectronic oscillator (OEO) [7]-[12]. An OEO uses a laser source that pumps an electrooptic modulator, the modulated light is detected by a photodetector and the electrical signal in turn drives the modulator. The optoelectronic feedback loop is essentially a microwave cavity, so oscillation is maintained when the open-loop gain reaches and saturates at unity. Highly stable oscillation is attained without an external reference by using a long optical delay line to increase the quality factor [12]. The optical spectrum usually consists of the main peak from the pump and two small sidebands from the modulator [9], although mode-locked operation can also be realized by incorporating the laser into the OEO loop [10], [11]. However, the work reported here is fundamentally different from an OEO in a few aspects. The optically injected laser in period-one dynamics is by itself a microwave oscillator. In this state, the microwave gain of the slave laser is already saturated even without the optoelectronic feedback. Instead of producing the microwave oscillation, the feedback loop provides the delayed replica of the microwave signal to injection lock the existing oscillation. The optical spectrum consists of two optical lines, 
which can be tuned in frequency separation and relative amplitude by adjusting the optical injection parameters. In particular, two coherently locked optical lines of equal amplitudes can be generated. As a result, the setup is believed to be complementary to the OEO in applications that require two strong optical lines.

Under different operating conditions, an optically injected semiconductor laser can be found in many different dynamical states, such as stable injection locking, period-one oscillation, period doubling, period quadrupling, and chaos [13], [14]. The period-one oscillation frequency increases with the injection strength [5], [14]. A semiconductor laser subject to optoelectronic feedback also have many different dynamical states, including stable oscillation, periodic pulsing, quasi-periodic pulsing, and chaotic pulsing [15], [16], which are very different from those of an optically injected laser. The combination of optical injection and optoelectronic feedback increases the complexity and enriches the nonlinear dynamics of a semiconductor laser [17]. Thus, it is entirely not straightforward to expect in advance that adding optoelectronic feedback to an optically injected semiconductor laser in period-one oscillation can stabilize the oscillation to narrow the microwave linewidth [19].

In this paper, we demonstrate experimentally through detailed, quantitative studies that the linewidth of a tunable microwave subcarrier generated by period-one oscillation of an optically injected semiconductor laser can be substantially reduced by proper optoelectronic feedback at a strength small enough not to drive the system into highly complex dynamical states. The microwave frequency is tuned in the range of $10-23 \mathrm{GHz}$ with its linewidth reduced from the range of 40-120 MHz without stabilization by three orders of magnitude to the range of $10-160 \mathrm{kHz}$ with stabilization through optoelectronic feedback alone. The effect of a small microwave modulation, both alone and in combination with the feedback, is also investigated. Following this introduction, the experimental setup for this study is described in Section II. The experimental results of linewidth narrowing by optoelectronic feedback, as well as detailed comparison of such results with those obtained by external modulation and a combination of optoelectronic feedback and modulation, are reported in Section III. This paper is concluded by a summary of this research in Section IV.

\section{EXPERIMENTAL SETUP}

The experimental setup is schematically shown in Fig. 1. The lasers used are single-mode distributed feedback (DFB) semiconductor lasers at $1.3-\mu \mathrm{m}$ wavelength. The master laser has a threshold of $15 \mathrm{~mA}$. It is biased at $112.5 \mathrm{~mA}$ and is temperature stabilized at $16.00^{\circ} \mathrm{C}$. The slave laser has a threshold of $18 \mathrm{~mA}$. It is biased at $40.00 \mathrm{~mA}$ and is temperature stabilized at $18.00{ }^{\circ} \mathrm{C}$. At this operating condition, the slave laser has a resonance oscillation frequency of $10.25 \mathrm{GHz}$ and a linewidth enhancement factor of 3.2, both of which are experimentally measured. Throughout the experiments reported in this paper, the detuning of the master laser frequency from the free-running frequency of the slave laser is fixed at $2.3 \mathrm{GHz}$, unless otherwise specified. Only the optical injection strength is varied to tune the

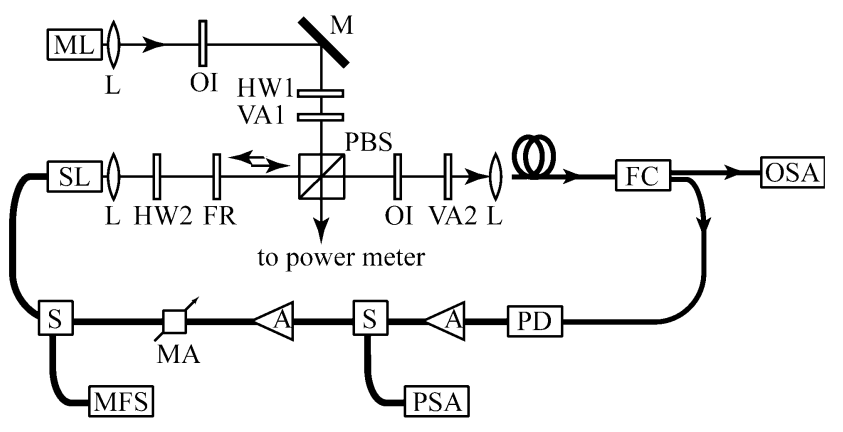

Fig. 1. Schematics of the experimental setup. ML: master laser; SL: slave laser; OI: optical isolator; HW: half-wave plate; VA: variable optical attenuator; PBS: polarizing beam splitter; FR: Faraday rotator; L: lens; M: mirror; FC: fiber coupler; OSA: scanning Fabry-Pérot optical spectrum analyzer; PD: high-speed photodiode; A: microwave amplifier; S: microwave power splitter; PSA: microwave spectrum analyzer; MA: variable microwave attenuator; MFS: microwave frequency synthesizer.

period-one oscillation frequency of the slave laser under optical injection.

The half-wave plates and the Faraday rotator are arranged such that the master laser can inject into the slave laser, while the slave laser output is completely transmitted through the polarizing beam splitter. A power meter is used to monitor the master laser residue $P_{i}$ when the injection strength is varied by the attenuator VA1. The normalized injection strength $\xi_{i}$ is proportional to the square root of $P_{i}$ because $\xi_{i}$ is defined with respect to the injection field amplitude [5]. A measured power of $P_{i}=1 \mu \mathrm{W}$ corresponds approximately to an injection strength of $\xi_{i}=0.0063$. The slave laser output is coupled into a fiber and is then detected by a high-speed photodetector of a $35-\mathrm{GHz}$ bandwidth. The current signal from the photodetector is amplified by two RF amplifiers with a $26.5-\mathrm{GHz}$ bandwidth and is then fed back to the slave laser through a bias tee. The optical spectrum is monitored by a scanning Fabry-Pérot interferometer that has a free-spectral range of $200 \mathrm{GHz}$ and a finesse greater than 5000. The RF power spectrum of the slave laser output is monitored by an HP E4407B RF spectrum analyzer that has a spectral range from $9 \mathrm{kHz}$ to $26.5 \mathrm{GHz}$. The feedback strength can be varied by the microwave attenuator and the optical attenuator VA2 for coarse and fine adjustment, respectively. The optoelectronic feedback power that is sent to the laser $P_{f}$ can be deduced from the microwave spectrum and the setting of the microwave attenuator. The normalized feedback strength $\xi_{f}$ is proportional to the output current of the detector [15]. Because the feedback electrical power $P_{f}$ is proportional to the square of the detector current, $\xi_{f} \propto \sqrt{P_{f}}$. At the given operating conditions, $P_{f}=0 \mathrm{dBm}$ approximately corresponds to $\xi_{f}=0.16$, and $P_{f}=-20 \mathrm{dBm}$ corresponds to $\xi_{f}=0.016$. The slave laser can also be modulated by a microwave frequency synthesizer that sends a microwave power of $P_{m}$ into the laser. The normalized modulation strength, $\xi_{m}$, is related to $P_{m}$ in the same way as $\xi_{f}$ is related to $P_{f}$.

As will be seen in the experimentally recorded power spectra discussed in Section III, the microwave spectral line has an irregular structure that is formed with fluctuating structures caused by the frequency jitter within the spectral line. Thus, it is not possible to quantify the linewidth using the common definition of full width at half-maximum, or 3-dB linewidth, 
because the spectral line does not have a clear shape, nor a steady peak, when its spectrum is taken with a sufficiently high resolution. To overcome this difficulty while having a common quantitative standard for comparing the linewidths of different spectra, we decide to account for the fluctuating nature of the spectral structure by measuring the spectral linewidth statistically using standard deviation. Thus, unless otherwise specified, the linewidth quoted in this paper is the standard deviation of the spectral power distribution, which is obtained by converting a power spectrum taken experimentally in the log scale into the linear scale and then calculating the standard deviation of its spectral distribution in linear scales.

\section{EXPERIMENTAL RESULTS}

\section{A. Optoelectronic Feedback}

The effect of optoelectronic feedback at a proper feedback strength that is able to significantly reduce the microwave linewidth of period-one oscillation of the optically injected slave laser is first studied. In this experiment, the detuning frequency between the master and slave lasers is fixed at $2.3 \mathrm{GHz}$ while the optical injection power from the master laser into the slave laser is varied by turning the variable optical attenuator VA1 in Fig. 1. The optoelectronic feedback loop, which includes the optical path and the electrical path in the loop, is fixed at a total effective path length of $10 \mathrm{~m}$ for a corresponding loop frequency of $30 \mathrm{MHz}$. The feedback strength is fixed at $\xi_{f} \approx 0.04$. The optoelectronic feedback can be switched on or off without affecting any other settings in the system. The external microwave source is switched off at all time so that the microwave subcarrier is generated entirely by the period-one oscillation of the slave laser, and the linewidth narrowing is caused completely by the optoelectronic feedback.

The optical spectra and the corresponding power spectra of the slave laser at different optical injection strengths with the optoelectronic feedback turned on or off are shown in Fig. 2. The optical spectra are shown in the left column, and the corresponding power spectra are shown in the right column. The center of all optical spectra at the zero frequency offset is set at the free-running optical frequency of the slave laser. The center of each power spectrum at the zero frequency is set at the frequency of the period-one microwave frequency, which varies as the optical injection strength is varied. The two traces in each frame are taken under the same experimental conditions with the optoelectronic feedback turned off for the upper trace and turned on for the lower trace.

For Fig. 2(a) and (b), the slave laser is free running without optical injection from the master laser. Thus, the upper trace in Fig. 2(a) shows its free-running optical spectrum without any external perturbation. The small peak at $-4.5 \mathrm{GHz}$ is due to the high-order transverse mode of the Fabry-Pérot optical spectrum analyzer and can be ignored. The power spectrum, which is centered at the relaxation resonance frequency of $10.25 \mathrm{GHz}$ for the free-running slave laser, appears flat for the narrow spectral range shown in Fig. 2(b) because it is a broad spectrum without a pronounced peak. When optoelectronic feedback is applied, both optical and power spectrum show no significant changes, as can be seen by comparing the lower traces in Fig. 2(a) and (b) to the upper traces. Thus, the optoelectronic feedback alone at

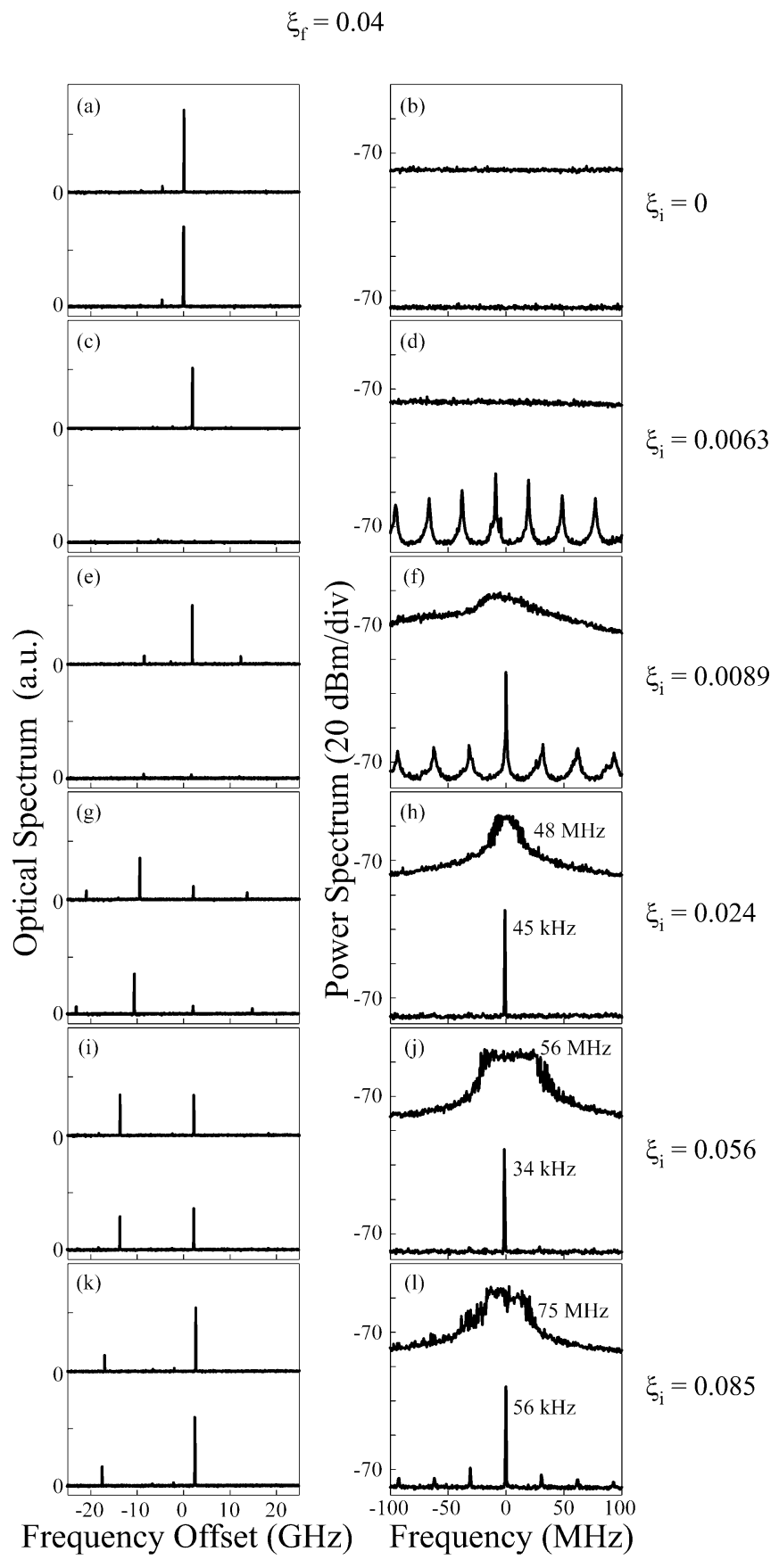

Fig. 2. Optical and power spectra of the slave laser at different injection strengths with the optoelectronic feedback turned on or off. The optical spectra are shown in the left column, and the corresponding power spectra are shown in the right column. The optical spectrum and power spectrum in each row are taken under the optical injection strength indicated for the row. The optical spectra are offset to the free-running slave laser frequency. The power spectra are centered at the respective peak frequencies. The two traces in each frame are taken under the same experimental conditions with the optoelectronic feedback turned off for the upper trace and turned on for the lower trace. The standard deviation linewidths are labeled.

the given strength is not strong enough to induce any interesting dynamics.

For Fig. 2(c) and (d), the slave laser is optically injected at $\xi_{i}=0.0063$. In the absence of optoelectronic feedback, the slave laser is stably injection locked by the master laser at the frequency offset of $2.3 \mathrm{GHz}$, as is seen in the upper trace of Fig. 2(c). Its power spectrum shown in the upper trace of 
Fig. 2(d) is again centered at the relaxation oscillation frequency with no significant structure. When the optoelectronic feedback is turned on, the dynamics of the slave laser is changed from stable locking to chaos. Energy is spread to a wide spectrum, as can be seen from the optical spectrum in the lower trace of Fig. 2(c). The corresponding power spectrum seen in the lower trace of Fig. 2(d) is only a small fraction of a broad spectrum that is characteristic of optical chaos.

As the injection strength is increased to $\xi_{i}=0.0089$ in the absence of optoelectronic feedback, the slave laser begins to enter period-one oscillation at the relaxation resonance frequency of $10.25 \mathrm{GHz}$. The optical spectrum and power spectrum of this state are shown in the upper traces of Fig. 2(e) and (f), respectively. The power spectrum shows that the period-one oscillation peak just begins to develop. When the optoelectronic feedback is applied, it drives the system again into chaos, resulting in the broad optical and power spectra seen, respectively, in the lower traces of Fig. 2(e) and (f). The power spectrum exhibits a repetitive structure with characteristic spectral peaks separating at $30 \mathrm{MHz}$ of the feedback loop frequency. Nevertheless, what is seen here is only a small section of the broad power spectrum spreading beyond $10 \mathrm{GHz}$. It is also observed that with the feedback still applied, a slight increment of the optical injection can drive the slave laser into period-two oscillation before it enters the period-one state. The period-doubling route and the characteristics of the broad power spectrum indicate that the state is chaotic.

Further increasing the optical injection power enhances the period-one oscillation and increases the oscillation frequency. Meanwhile, the effect of optoelectronic feedback changes from inducing chaos to stabilizing the oscillation by narrowing the microwave frequency of the period-one oscillation. These scenarios are demonstrated in optical and power spectra shown in Fig. 2(g)-(l) for three different injection strengths of $\xi_{i}=$ $0.024,0.056$, and 0.085 , respectively. From the optical spectra shown in Fig. 2(g), (i), and (k), it is seen that the slave laser remains locked by the master laser at a detuning frequency of $2.3 \mathrm{GHz}$ while the period-one oscillation frequency varies from 12 to 16 to $20 \mathrm{GHz}$ as the injection power increases successively through the three levels. The amplitudes of the optical spectral lines also vary as the injection power varies. Very little change is observed between the upper and lower traces of each optical spectral frame, indicating that the dynamical state of the slave laser is not much affected by the optoelectronic feedback. However, significant changes are seen in the power spectra shown in Fig. 2(h), (j), and (l), where linewidth narrowing by three orders of magnitude due to optoelectronic feedback is clearly demonstrated by comparing the upper and lower traces of each frame. These general characteristics remain effective as the optical injection power is further increased as long as the slave laser remains in the period-one state. Thus, it is demonstrated that a fixed optoelectronic feedback loop at a fixed strength can be used to stabilize the period-one oscillation with a significant reduction in the microwave linewidth while the oscillation frequency is tuned by varying an optical injection parameter such as the injection strength.

The tunability of the microwave frequency and the linewidth narrowing effect of the optoelectronic feedback are summarized in Fig. 3(a) and (b), respectively, where the open circles are data taken with the feedback turned off and the closed circles

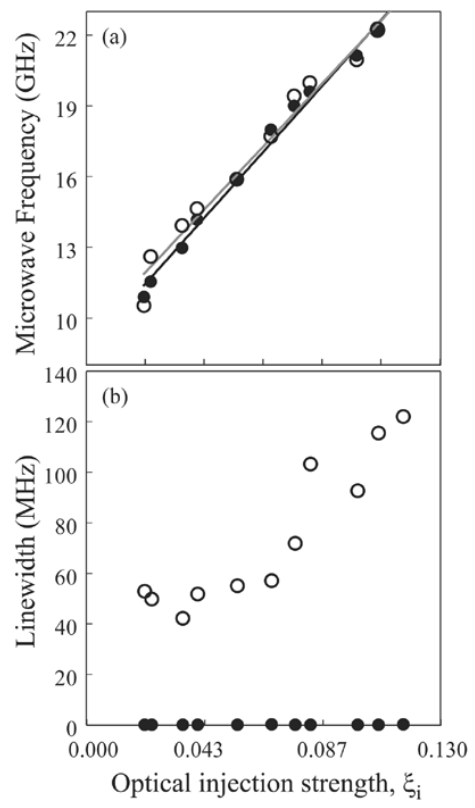

Fig. 3. Microwave frequency and standard deviation linewidth versus optical injection strength $\xi_{i}$. The open and closed symbols are data taken with the feedback turned off and on, respectively. The gray and dark curves in (a) are the linear regressions for the data shown as open and closed circles, respectively. The data points in (b) for the narrowed microwave linewidth in the presence of the feedback are all below $160 \mathrm{kHz}$ and cannot be resolved in this plot.

are data taken with the feedback turned on. It is clearly seen in Fig. 3(a) that the tunability of the microwave frequency is completely not affected by the optoelectronic feedback over the entire range where the slave laser remains in the period-one state. By contrast, Fig. 3(b) shows that the microwave linewidth is significantly reduced by the optoelectronic feedback while the oscillation frequency is unchanged. The data points in Fig. 3(b) for the narrowed microwave linewidth in the presence of the feedback cannot be resolved in this plot because they fall below $160 \mathrm{kHz}$.

\section{B. Feedback Strength}

To study the dependence of the linewidth narrowing effect on the optoelectronic feedback strength, the feedback strength is varied while the slave laser is optically injected at a fixed strength of $\xi_{i}=0.056$ and a frequency detuning of $2.3 \mathrm{GHz}$ for the period-one state that has the spectra shown in Fig. 2(i) and (j). In this period-one state, the microwave frequency is $16 \mathrm{GHz}$ and the optical spectrum show two spectral lines of almost equal amplitudes at the spacing of $16 \mathrm{GHz}$, as is seen in Fig. 2(i).

With all other operating conditions staying fixed, the feedback strength is gradually increased from zero. Fig. 4 shows three power spectra that represent the quantitative sequence of the linewidth narrowing effect by optoelectronic feedback. For Fig. 4(a), no feedback is applied; the spectrum is just that of the period-one oscillation generated by optical injection alone. When a small feedback of $\xi_{f}=0.008$ is applied, a spectrum of many equally spaced narrow lines, each of about $160-\mathrm{kHz}$ linewidth, as shown in Fig. 4(b), is observed. The frequency spacing in this spectrum is the feedback loop frequency of $30 \mathrm{MHz}$ for the effective loop path length of $10 \mathrm{~m}$ when this spectrum is taken. To verify this characteristic, the 


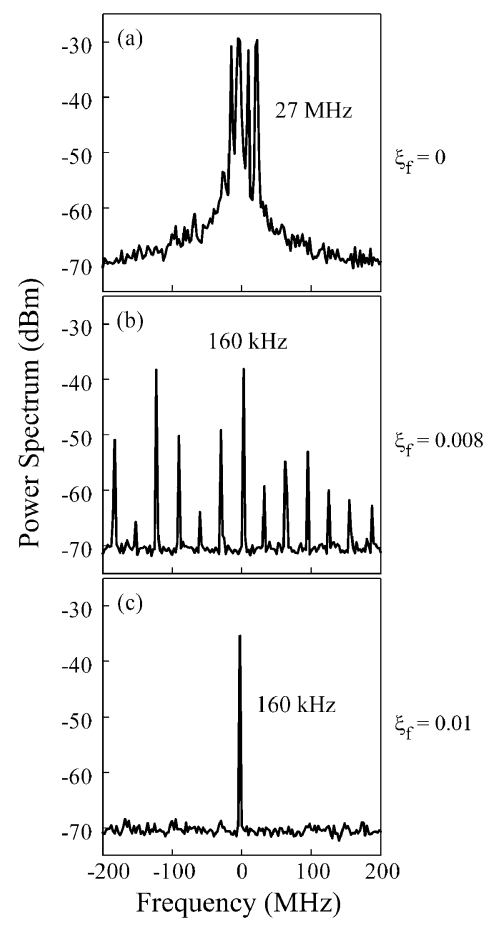

Fig. 4. Power spectra centered at the peak microwave frequency of the period-one oscillation of the optically injected slave laser for different feedback strengths as labeled. The standard deviation linewidths are labeled.

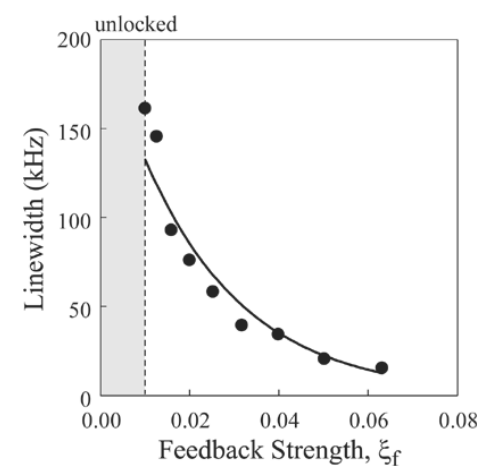

Fig. 5. Standard deviation linewidth as a function of optoelectronic feedback strength, $\xi_{f}$. The locking threshold is $\xi_{f}=0.01$. The curve is the exponential regression of the experimental data shown as solid circles.

feedback loop length is varied over the range of 7-15 $\mathrm{m}$, limited by our experimental setting. It is found that the frequency spacing in this spectrum changes accordingly. Clearly, at this low feedback strength, the optoelectronic feedback is able to narrow the microwave oscillation linewidth but is not sufficient to lock it and suppress competing mode frequencies from the feedback loop itself. As shown in Fig. 4(c), locking of the period-one oscillation frequency by suppressing the side mode frequencies is accomplished at a threshold feedback strength of $\xi_{f}=0.01\left(P_{f}=-24 \mathrm{dBm}\right)$. At this threshold feedback strength, the microwave linewidth is narrowed to about $160 \mathrm{kHz}$. When the feedback strength is increased, the microwave frequency remains stably locked with a decreasing linewidth. The experimentally measured linewidth as a function of the feedback strength $\xi_{f}$ is shown in Fig. 5. It is seen that the linewidth is narrowed to a range between 10 and $160 \mathrm{kHz}$, depending on the feedback strength. The trend continues even

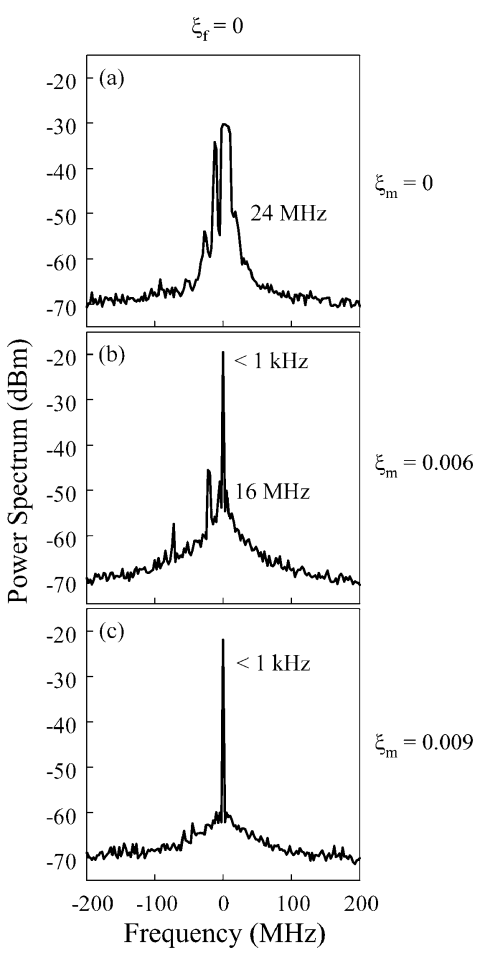

Fig. 6. Power spectra centered at the peak microwave frequency of the period-one oscillation of the optically injected slave laser for different external modulation powers as labeled. The threshold for stable locking is $\xi_{m}=0.009$, shown in (c). The standard deviation linewidths are labeled. The linewidth in (c) cannot be resolved by the $1 \mathrm{kHz}$ resolution limit of the instrument.

in the unlocked range, but the side modes are not suppressed, as in Fig. 4(b). Note that the narrowed linewidth is actually determined by the broadening due to the frequency jitter within the linewidth. Thus, the data in Fig. 5 indicate that the optoelectronic feedback stabilizes the microwave oscillation by suppressing the frequency jitter with increased effectiveness at an increased feedback strength.

\section{External Modulation}

In order to further reduce the linewidth, an external microwave source can be used to slightly modulate the slave laser. As is demonstrated by Simpson and Doft [1], the resulting linewidth can be narrower than $1 \mathrm{kHz}$. To study the effect of an external modulation on our system, the same procedure is first repeated here. The experimental results are then used to compare with those obtained with both external modulation and optoelectronic feedback. In this experiment, the slave laser is optically injected at a fixed strength of $\xi_{i}=0.056$ and a frequency detuning of $2.3 \mathrm{GHz}$ for the same period-one state studied above that has the spectra shown in Fig. 2(i) and (j). The external microwave modulation frequency is fixed at $16 \mathrm{GHz}$ in tune with the period-one oscillation frequency.

Fig. 6(a)-(c) shows three power spectra of the slave laser taken at three different external microwave modulation strengths of $\xi_{m}=0,0.006$, and 0.009 , respectively, while the optoelectronic feedback is completely turned off. It is found that the threshold modulation strength to completely lock the microwave oscillation to a stable narrow line is $\xi_{m}=0.009\left(P_{m}=-25 \mathrm{dBm}\right)$, corresponding to that of Fig. 6(c). Above this threshold, the line remains stably locked 


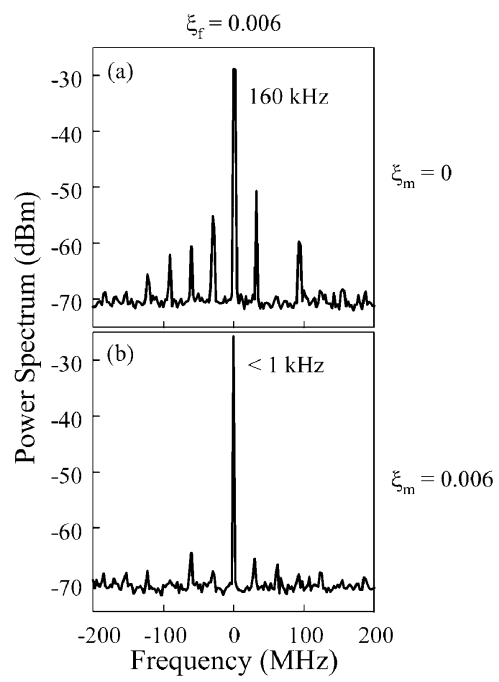

Fig. 7. Power spectra centered at the peak microwave frequency of the period-one oscillation of the optically injected slave laser with the optoelectronic feedback fixed at $\xi_{f}=0.006$ and the external modulation power turned (a) off and (b) on at $\xi_{m}=0.006$.

with a narrow linewidth below our instrument resolution limit of $1 \mathrm{kHz}$. Below this threshold, the modulation partially locks the oscillation to a narrow central peak, also below our resolution limit of $1 \mathrm{kHz}$, but it is not strong enough to stably lock the oscillation. In this situation, the period-one oscillation drifts in and out of locking by the microwave modulation, resulting in a jittering broad linewidth of about $16 \mathrm{MHz}$ seen superimposed with the central narrow line of less than $1 \mathrm{kHz}$ in Fig. 6(b).

The combined effect of external modulation and optoelectronic feedback is studied by setting the feedback strength at a fixed low level of $\xi_{f}=0.006$ while $\xi_{m}$ is varied. Note that according to the experiment done for the data shown in Fig. 5, this feedback strength is below the locking threshold for suppressing the side mode frequencies of the feedback loop. The power spectrum shown in Fig. 7(a) for $\xi_{m}=0$ without the external modulation clearly shows the characteristic of many side frequencies equally spaced at $30 \mathrm{MHz}$. The peak in this spectrum has a linewidth of $160 \mathrm{kHz}$. When a weak external modulation of $\xi_{m}=0.006$ is applied, the side modes are suppressed and the oscillation is stably locked with the locked microwave frequency narrowed to a linewidth below our resolution limit of $1 \mathrm{kHz}$, as shown in Fig. 7(b). Note also that this modulation power of $\xi_{m}=0.006$ alone is below the threshold to stably lock the oscillation, as seen in Fig. 6 and discussed above. Thus, this study shows that the external modulation and the optoelectronic feedback together have a positively enhancing effect that reduces the stable locking threshold for each other.

\section{Linewidth Narrowing Effects}

So far, it is clearly demonstrated that proper optoelectronic feedback also can reduce the microwave linewidth of a period-one oscillation by three orders of magnitude. An accurately tuned external modulation, either alone or in combination with optoelectronic feedback, can further reduce the linewidth. An experiment is carried out to study the detailed characteristics of the linewidth narrowing by optoelectronic
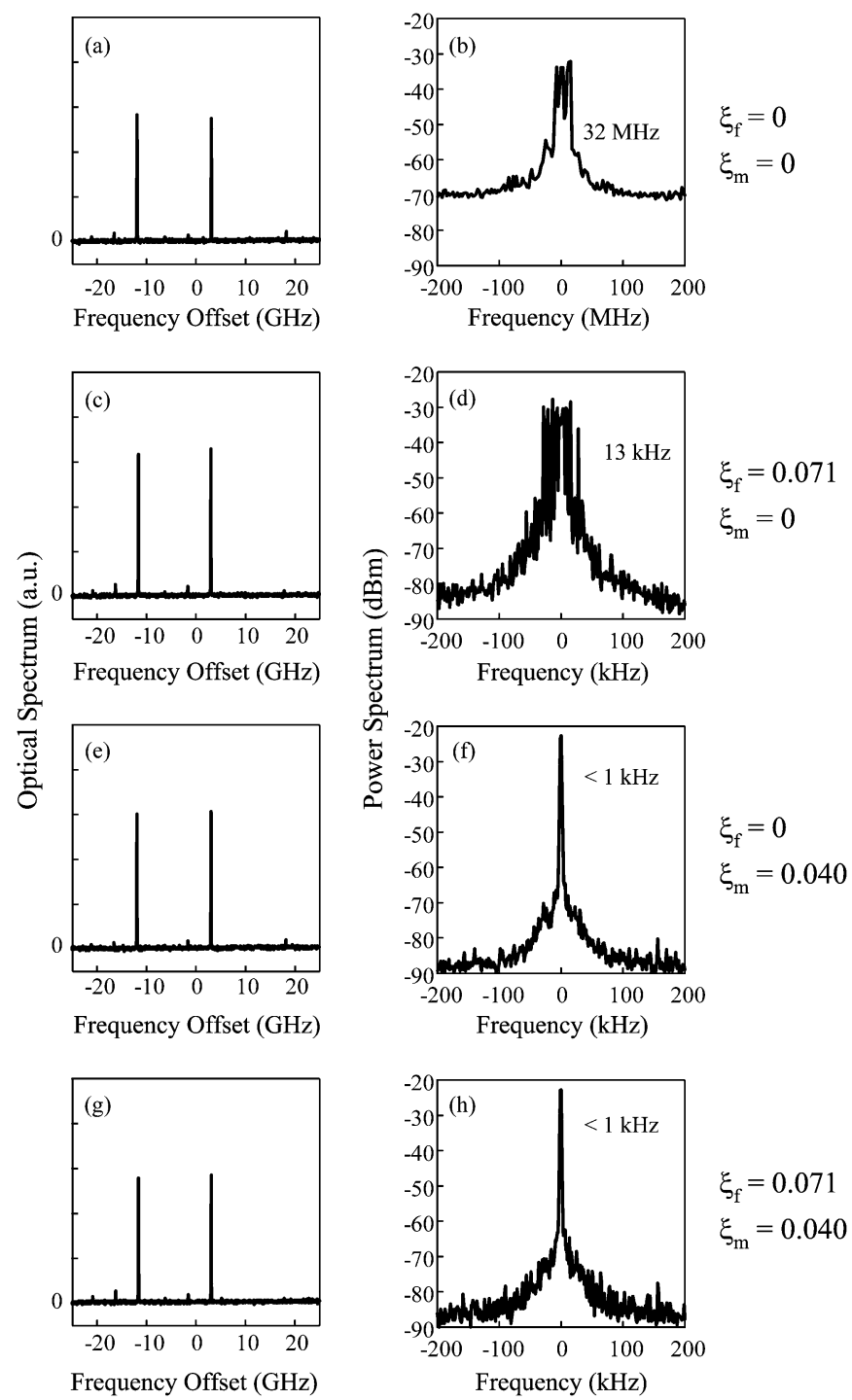

Fig. 8. Optical and power spectra of the slave laser at a fixed optical injection strength of $\xi_{i}=0.056$ with (a) and (b) optoelectronic feedback and external modulation both turned off, (c) and (d) feedback on but modulation off, (e) and (f) feedback off but modulation on, and (g) and (h) feedback and modulation both on at the strengths labeled. The optical spectra are shown in the left column, and the corresponding power spectra are shown in the right column. The optical spectra are offset to the free-running slave laser frequency. The power spectra are centered at the respective peak frequencies. The standard deviation linewidths are labeled.

feedback and external modulation. In this experiment, the slave laser is again optically injected at a fixed strength of $\xi_{i}=0.056$ and a frequency detuning of $2.3 \mathrm{GHz}$ for the same period-one state studied above. The external microwave modulation frequency is again fixed at $16 \mathrm{GHz}$ in tune with the period-one oscillation frequency. The feedback is fixed at $\xi_{f}=0.071\left(P_{f}=-7 \mathrm{dBm}\right)$ and a loop length of $10 \mathrm{~m}$, and the modulation is fixed at $\xi_{m}=0.040\left(P_{m}=-12 \mathrm{dBm}\right)$. These feedback and modulation powers are both above their respective thresholds to stably lock the microwave frequency of the period-one oscillation into a narrow line. The feedback and the modulation can be turned on or off independently.

Fig. 8 shows the optical spectra, in the left column, and the power spectra, in the right column, of the slave laser output 


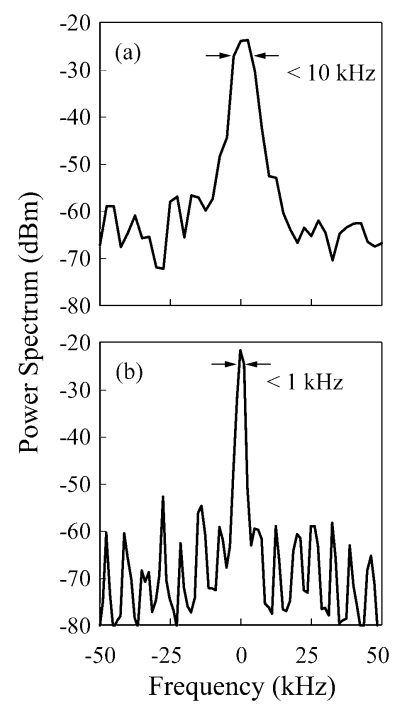

Fig. 9. Power spectrum of the period-one microwave frequency of the optically injected slave laser: (a) with optoelectronic feedback only at $\xi_{f}=0.071$ and (b) with external modulation only at $\xi_{m}=0.071$. The resolution bandwidths are $10 \mathrm{kHz}$ and $1 \mathrm{kHz}$ for (a) and (b), respectively. The 3-dB linewidths are below the respective resolution bandwidths.

taken under the same experimental conditions but with the feedback and the modulation turned on or off in four different combinations. First, it is seen that the four optical spectra are almost identical, indicating that the period-one dynamic state of the slave laser is not affected by the turning on or off of the feedback and the modulation. Indeed, any small differences among these four optical spectra are caused by the ever-present constant fluctuations of the system. The small peaks seen at the baselines of these spectra are high-order transverse modes of the scanning Fabry-Pérot interferometer and can be ignored. Significant differences are clearly seen in the power spectra taken under different feedback and modulation conditions. In Fig. 8(b), with both feedback and modulation turned off, a large microwave linewidth measured to be $32 \mathrm{MHz}$ is recorded. For Fig. 8(d), the feedback alone is turned on to reduce the microwave linewidth by more than three orders of magnitude to $13 \mathrm{kHz}$, which is still mainly determined by the frequency jitter. In Fig. 8(f), it is seen that the line is narrowed to below the resolution limit of $1 \mathrm{kHz}$ by turning the external modulation on alone. Because the external modulation is significantly above its locking threshold, the power spectrum seen in Fig. 8(h) taken with both feedback and modulation turned on remains essentially the same as that shown in Fig. 8(f). Combined with the data shown in Fig. 7 and discussed above, the spectra shown in Fig. 8(f) and (h) indicate that under proper operating conditions the combination of feedback and modulation does not have negative effects. If the modulation frequency is properly tuned, feedback and modulation enhance each other near their thresholds but do not compete with each other to drive the laser into undesirable dynamics when they are both high above threshold.

As shown in Fig. 5, it is possible to reduce the linewidth by optoelectronic feedback alone to the order of $10 \mathrm{kHz}$. It seems possible to further reduce the linewidth without an external modulation. The power spectrum shown in Fig. 9(a), which is taken with optoelectronic feedback alone at $\xi_{f}=0.071$, clearly shows this possibility. This spectrum is taken with the spectral

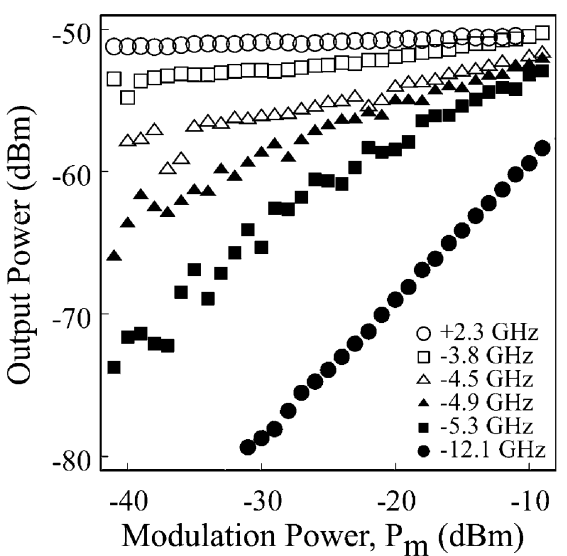

Fig. 10. Microwave output power versus modulation power when the optoelectronic feedback loop is disconnected. Data for different optical injection detunings are presented. Open and closed symbols represent period-one and stable optical locking dynamics, respectively.

resolution of the spectrum analyzer set at $10 \mathrm{kHz}$. Its $3-\mathrm{dB}$ linewidth is limited by this resolution limit, indicating that it has a linewidth less than $10 \mathrm{kHz}$. Unfortunately, we failed to take this spectrum with the best resolution of our instrument at $1 \mathrm{kHz}$ because this line drifts away within the long scanning time of the spectrum analyzer at the $1-\mathrm{kHz}$ resolution. When an external microwave modulation at $\xi_{m}=0.071$ is applied, the spectrum remains steady to allow the slow spectral scan at the $1-\mathrm{kHz}$ resolution regardless of whether the feedback is applied or not. The result is shown in Fig. 9(b), which shows a narrow line with a 3-dB linewidth again limited by the resolution limit of $1 \mathrm{kHz}$.

In order to have a better understanding on the linewidth-narrowing mechanism, the microwave open-loop gain is examined by disconnecting the optoelectronic feedback. The optical injection strength is set at $\xi_{i}=0.056$, and an external modulation of power $P_{m}$ at the period-one oscillation frequency is sent into the slave laser. The output microwave power after the photodetector is monitored while $P_{m}$ increases. The result is shown in Fig. 10. At the optical detuning of $2.3 \mathrm{GHz}$, as in the previous experiments, the output power is saturated. The microwave gain is saturated even for $P_{m}$ below the locking threshold of about $-25 \mathrm{dBm}$ for external modulation or optoelectronic feedback. It shows that the slave laser already acts as a microwave oscillator at the period-one frequency with a gain that is saturated by the period-one oscillation before the optoelectronic feedback is applied. The optoelectronic feedback loop does not form a microwave oscillator, but it filters and injects the microwave to lock the slave laser. This explains the linewidth narrowing effect. A similar self-injection locking phenomenon is observed in other oscillatory systems [12]. The experiment is repeated for different optical injection detunings. As the detuning decreases from 2.3 to $-4.5 \mathrm{GHz}$, the slave laser stays in the period-one state, but the microwave gain saturation decreases as the Hopf bifurcation line approaches. The bifurcation line is crossed between -4.5 and $-4.9 \mathrm{GHz}$, while the slave laser exits the period-one state and enters into stable locking state [5]. The microwave gain saturation decreases further as the detuning decreases to $-5.3 \mathrm{GHz}$. At $-12.1 \mathrm{GHz}$, the slave laser is well within the stable locking region, and the modulation response is similar to that of a free-running laser with enhanced 
bandwidth and has a linear gain [18]. In the stable locking region, the optoelectronic feedback can be reintroduced to form an OEO. Oscillation is observed when the open-loop gain is increased to unity using the RF amplifiers. Thus, the period-one linewidth-narrowing effect studied in this paper is due to self-injection locking, but an OEO can also be formed when the laser exits the period-one region and enters into the stable locking region.

\section{CONCLUSION}

The generation of tunable narrow-linewidth microwave on an optical wave is experimentally demonstrated on an optically injected semiconductor laser in the period-one dynamical state with optoelectronic feedback. The microwave carried by the optical wave generated from an optically injected slave semiconductor laser is tuned in the range of $10-23 \mathrm{GHz}$ by varying the strength of optical injection at a fixed detuning frequency between the master and slave lasers. Based on our nonoptimized apparatus, the microwave linewidth is reduced from the range of 40-120 MHz without stabilization by three orders of magnitude to the range of $10-160 \mathrm{kHz}$ with stabilization through optoelectronic feedback alone. The narrow linewidth under the stabilization of optoelectronic feedback is mainly determined by the frequency jitter. Such jitter decreases with the strength of optoelectronic feedback. The effect of a small microwave modulation is also investigated. It reduces the linewidth to below the $1 \mathrm{kHz}$ resolution limit of our instrument, and the modulation power required is decreased by the optoelectronic feedback.

The technique of generating a broadly tunable narrowlinewidth microwave subcarrier on an optical wave by properly combining optical injection and optoelectronic feedback as studied in this paper exploits the nonlinear dynamics of the semiconductor lasers without the need of any external microwave source. To the best of our knowledge, the broadly tunable linewidth narrowing by optoelectronic feedback is a new nonlinear dynamical phenomenon. Further experimental and theoretical studies along the line of microwave self-injection locking are needed to better understand this phenomenon and to explore the possibility of other similar phenomena, as well as to connect this phenomenon to other complex dynamical states of a semiconductor laser subject to both optical injection and optoelectronic feedback.

\section{ACKNOWLEDGMENT}

The authors would like to thank Drs. T. B. Simpson and S.-K. Hwang for their helpful discussions.

\section{REFERENCES}

[1] T. B. Simpson and F. Doft, "Double-locked laser diode for microwave photonics applications," IEEE Photon. Technol. Lett., vol. 11, pp. 1476-1478, Nov. 1999.

[2] U. Gliese, T. N. Nielsen, M. Bruun, E. L. Christensen, K. E. Stubkjær, S. Lindgren, and B. Broberg, "A wideband heterodyne optical phase-locked loop for generation of 3-18 GHz microwave carriers," IEEE Photon. Technol. Lett., vol. 4, pp. 936-938, Aug. 1992.

[3] J. Genest, M. Chamberland, P. Tremblay, and M. Tetu, "Microwave signals generated by optical heterodyne between injection-locked semiconductor lasers," IEEE J. Quantum Electron., vol. 33, pp. 989-998, June 1997.

[4] L. A. Johansson and A. J. Seeds, "Generation and transmission of millimeter-wave data-modulated optical signals using an optical injection phase-lock loop," J. Lightwave Technol., vol. 21, pp. 511-520, Feb. 2003.
[5] T. B. Simpson, J. M. Liu, and A. Gavrielides, "Small-signal analysis of modulation characteristics in a semiconductor laser subject to strong optical injection," IEEE J. Quantum Electron., vol. 32, pp. 1456-1468, Aug. 1996.

[6] T. B. Simpson, "Phase-locked microwave-frequency modulations in optically-injected laser diodes," Opt. Commun., vol. 170, no. 1-3, pp. 93-99, 1999.

[7] X. S. Yao and L. Maleki, "Optoelectronic microwave oscillator," J. Opt. Soc. Amer. B, vol. 13, no. 8, pp. 1725-1735, 1996.

[8] M. F. Lewis, "Novel RF oscillator using optical components," Electron. Lett., vol. 28, no. 1, pp. 31-32, 1992.

[9] A. B. Matsko, L. Maleki, A. A. Savchenkov, and V. S. Ilchenko, "Whispering gallery mode based optoelectronic microwave oscillator," J. Mod. Opt., vol. 50, no. 15-17, pp. 2523-2542, 2003.

[10] X. S. Yao and L. Maleki, "Dual microwave and optical oscillator," Opt. Lett., vol. 22, no. 24, pp. 1867-1869, 1997.

[11] X. S. Yao, L. Maleki, and L. Davis, "Coupled opto-electronic oscillators," in Proc. 1998 IEEE Int. Frequency Control Symp., 1998, pp. $540-544$.

[12] X. S. Yao and L. Maleki, "Optoelectronic oscillator for photonic systems," IEEE J. Quantum Electron., vol. 32, pp. 1141-1149, July 1996.

[13] S. K. Hwang and J. M. Liu, "Dynamical characteristics of an optically injected semiconductor laser," Opt. Commun., vol. 183, no. 1-4, pp. 195-205, 2000.

[14] T. B. Simpson, J. M. Liu, K. F. Huang, and K. Tai, "Nonlinear dynamics induced by external optical injection in semiconductor lasers," Quantum Semiclass. Opt., vol. 9, no. 5, pp. 765-784, 1997.

[15] S. Tang and J. M. Liu, "Chaotic pulsing and quasi-periodic route to chaos in a semiconductor laser with delayed opto-electronic feedback," IEEE J. Quantum Electron., vol. 37, pp. 329-336, Mar. 2001.

[16] F. Y. Lin and J. M. Liu, "Nonlinear dynamics of a semiconductor laser with delayed negative optoelectronic feedback," IEEE J. Quantum Electron., vol. 39, pp. 562-568, Apr. 2003.

[17] —, "Nonlinear dynamical characteristics of an optically injected semiconductor laser subject to optoelectronic feedback," Opt. Commun., vol. 221, no. 1-3, pp. 173-180, 2003.

[18] S. K. Hwang, J. M. Liu, and J. K. White, "35-GHz intrinsic bandwidth for direct modulation in $1.3-\mu \mathrm{m}$ semiconductor lasers subject to strong injection locking," IEEE Photon. Technol. Lett., vol. 16, pp. 972-974, Apr. 2004.

[19] P. Saboureau, J.-P. Foing, and P. Schanne, "Injection-locked semiconductor lasers with delayed optoelectronic feedback," IEEE J. Quantum Electron., vol. 33, pp. 1582-1591, Sept. 1997.

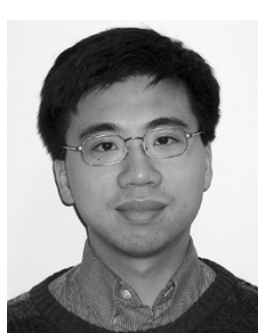

Sze-Chun Chan (S'98) received the B.Eng. degree in electrical and electronic engineering from the University of Hong Kong, Hong Kong, China, in 2001. $\mathrm{He}$ is currently working toward the M.S. and Ph.D. degrees in photonics at the University of California, Los Angeles.

His current research focuses on the nonlinear dynamics of semiconductor lasers and its applications.

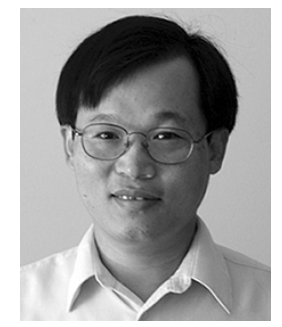

Jia-Ming Liu (M'83-SM'85) received the B.S. degree in electrophysics from National Chiao Tung University, Hsinchu, Taiwan 300, R.O.C., in 1975, and the S.M. and Ph.D. degrees in applied physics from Harvard University, Cambridge, MA, in 1979 and 1982, respectively.

He was an Assistant Professor in the Department of Electrical and Computer Engineering, State University of New York, Buffalo, from 1982 to 1983 and was a Senior Member of the Technical Staff with GTE Laboratories, Inc., from 1983 to 1986 . He is currently Professor of Electrical Engineering at the University of California, Los Angeles. His current research interests include development and application of ultrafast wavelength-tunable laser pulses, nonlinear and ultrafast processes in materials and devices, optical wave propagation, optical communications, nonlinear dynamics of lasers, and chaotic communications.

Dr. Liu is a Fellow of the Optical Society of America and the American Physical Society, a Senior Member of the IEEE Laser and Electro-Optics Society, and a Founding Member of the Photonics Society of Chinese-Americans. He became a Licensed Professional Electrical Engineer in 1977. 